ACCEPTED FOR PUBlication IN ApJ

Preprint typeset using $\mathrm{LTT}_{\mathrm{E} X} \mathrm{X}$ style emulateapj v. 5/2/11

\title{
LENTICULAR GALAXY IC 719: CURRENT BUILDING OF THE COUNTERROTATING LARGE-SCALE STELLAR DISK.
}

\author{
Ivan Yu. Katkov ${ }^{1}$, Olga K. Sil'Chenko ${ }^{1}$, Victor L. Afanasiev ${ }^{2}$ \\ Accepted for publication in ApJ
}

\begin{abstract}
We have obtained and analyzed long-slit spectral data for the lenticular galaxy IC 719. In this gas-rich S0 galaxy, its large-scale gaseous disk counterrotates the global stellar disk. Moreover in the IC 719 disk we have detected a secondary stellar component corotating the ionized gas. By using emission-line intensity ratios, we have proved the gas excitation by young stars and so are claiming current star formation, most intense in a ring-like zone at the radius of $10^{\prime \prime}(1.4 \mathrm{kpc})$. The oxygen abundance of the gas in the starforming ring is about half of the solar abundance. Since the stellar disk remains dynamically cool, we conclude that smooth prolonged accretion of the external gas from a neighboring galaxy provides current building of the thin large-scale stellar disk.
\end{abstract}

Subject headings: galaxies: elliptical and lenticular — galaxies: ISM — galaxies: kinematics and dynamics — galaxies: evolution — galaxies: individual: IC 719.

\section{INTRODUCTION}

Lenticular galaxies represent rather common morphological type in the nearby Universe: they constitute up to $15 \%$ of all galaxies in the field (Naim et al. 1995) being at the second place after spirals. Though they are more frequent in clusters, there is also a considerable population of quite isolated lenticulars (Sulentic et al. 2006), or of lenticulars in isolated pairs and triplets. Lenticulars are thought to lack gas in general; however just in the field the cold neutral hydrogen is found in up to $25 \%-45 \%$ of all lenticulars, by the contrast with the cluster members (Chamaraux et al. 1987; Grossi et al. 2009; Serra et al. 2012). The difference in the gas detection frequency depending on environment density is sometimes explained by numerous mechanisms of gas removing from the large-scale disks which are effective only in dense environments. But this scenario cannot explain the difference in gas kinematics depending on environment density: in the cases when the gas is found in cluster lenticulars, its rotation matches usually the rotation of the stellar components, while in the field S0s the gas kinematics is very often decoupled from that of the stellar components (Davis et al. 2011). Probably, the main difference related to the environments must be looked for in the area of the gas acquisition conditions, and not in the area of its subsequent dynamical evolution. Davis et al. (2011) suggest that the gas in cluster lenticulars is provided by the mass loss of the stellar components, and so comes from an intrinsic source. Field lenticulars are evidently feeded by external accretion; but the sources of the external accretion are not often recognized unambiguously. There are two main mechanisms that have been suggested by theorists, and to make choice between them - minor mergers or cold accretion from large-scale filaments - we need particular observational investiga-

\footnotetext{
katkov.ivan@gmail.com, olga@sai.msu.su,vafan@sao.ru

${ }^{1}$ Sternberg Astronomical Institute, M.V. Lomonosov Moscow State University, Moscow, 119992 Russia

2 Special Astrophysical Observatory, Russian Academy of Sciences, Nizhnii Arkhyz, Karachaevo-Cherkesskaya Republic, 369167 Russia
}

tions 'in depth'. So every new S0 galaxy with a significant kinematically decoupled gas component attracts close attention of investigators.

Though small amounts of kinematically decoupled gas are common in lenticular galaxies settling in low-density environments (Bertola et al. 1992), large counterrotating gaseous disks are rare, as well as gas-rich $\mathrm{S} 0$ galaxies at all. In the (R)SA(rs)0+ galaxy NGC 3626 (Ciri et al. 1995; Garcia-Burillo et al. 1998; Haynes et al. 2000), located at the periphery of a massive X-ray detected group, all the gas found, including ionized, molecular, and neutral species, counterrotates the stellar component. In unbarred galaxies and also group members, in SA0/a-galaxy NGC 3593 (Bertola et al. 1996) and in the SA(r)0+ galaxy NGC 4138 (Thakar et al. 1997) their counterrotating gas is already partly processed into stars, so these galaxies demonstrate two counterrotating stellar disks one of which rotates together with their gas. In the SA0 NGC 1596 (Chung et al. 2006) the outer gas, freshly accreted from the neighboring NGC 1602, counterrotates the inner part of the lenticular galaxy: the gas radial inflow in this unbarred galaxy is perhaps ineffective. To this list we have recently added two more lenticular galaxies with extended counterrotating gas, NGC 2551 and NGC 5631 (Sil'chenko et al. 2009); in the former one the GALEX detects also a broad ring of star formation in the disk-dominated area, in the latter we found two counterrotating stellar components. Interestingly, all the galaxies mentioned above are unbarred though some of them possess rings and some reveal signatures of oval distortion (Afanasiev \& Sil'chenko 2002; Sil'chenko et al. 2010) which may be traces of past interaction.

In the present paper, to the small list of the S0s with extended counterrotating gas, and to even smaller list of S0s with two counterrotating stellar components (Kuijken et al. 1996), we are adding one more interesting object. We have undertaken an analysis of various spectral data for the lenticular galaxy IC 719. The galaxy is of intermediate luminosity and constitutes an isolated non-interacting pair with IC 718 - a late-type galaxy of 

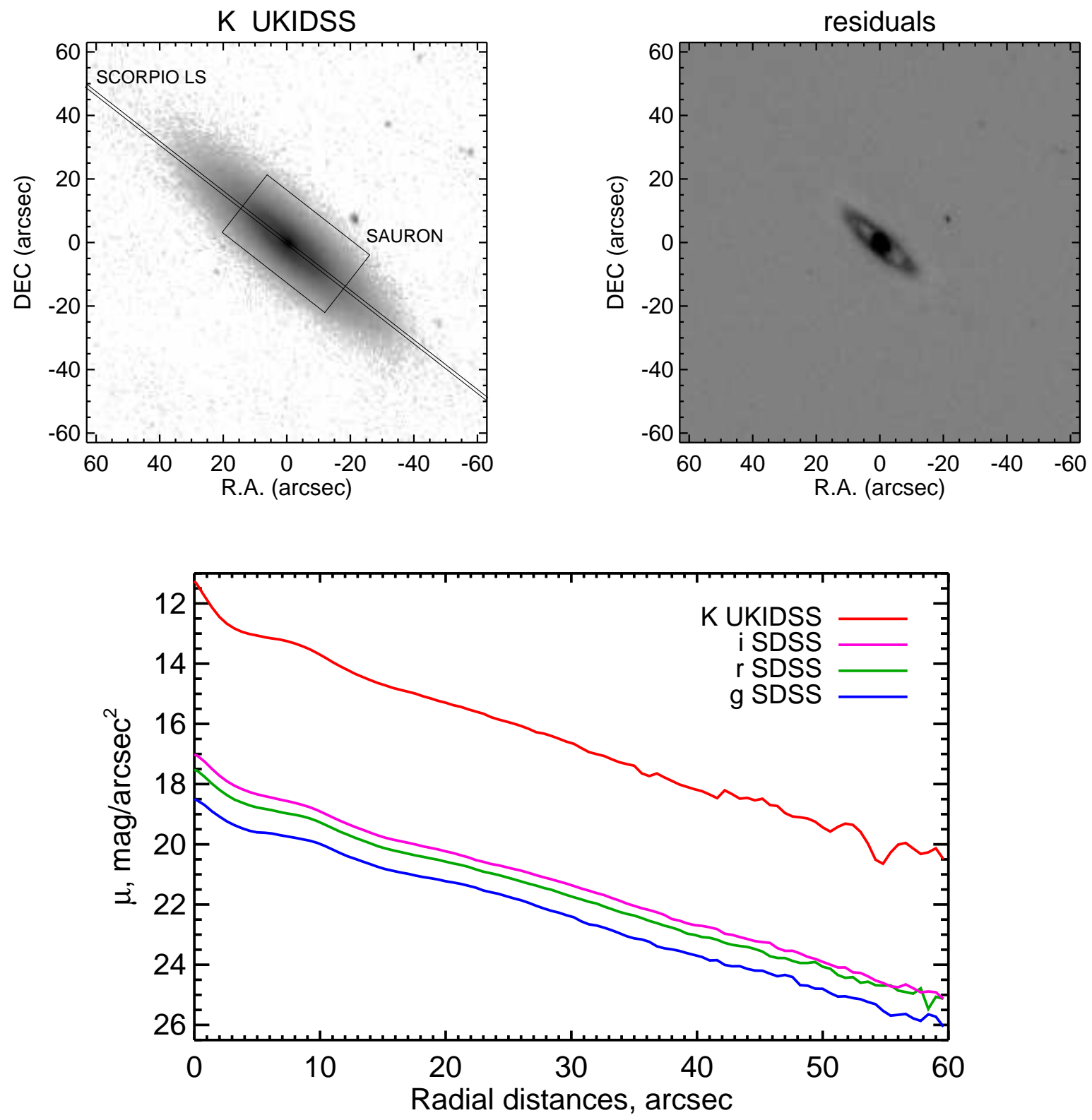

FIG. 1. - S0 galaxy IC 719: top left - a $K$-band image from the UKIDSS DR8; top right - residuals between $K$-band image and outward exponential disk model; bottom - the azimuthally averaged surface brightness profiles calculated from the SDSS $g, r, i$-band images and the UKIDSS $K$-image.

the similar luminosity. General characteristics of IC 719 are presented in the Table 1 , and the general view can be seen in Fig. 1. Though the galaxy is classified as an earlytype one in all catalogues and databases, one can assured that it is almost bulgeless: its surface brightness profiles represent a pure quasi-exponential disk (Fig. 1). The galaxy is rather gas-rich for the S0 type; moreover, neutral $\mathrm{HI}$ and molecular gas both are detected. The galaxy was included into the sample of the survey ATLAS-3D (Cappellari et al. 2011) and observed with the integralfield spectrograph SAURON (Bacon et al. 2001); as a result, the counterrotation of the gaseous and stellar components has been detected for its central part, $R<20^{\prime \prime}$. Meantime, the HI disk of IC 719 is very extended - much more extended than even its stellar disk (Grossi et al.
2009); however the large-scale velocity field of the HI is unknown. It would be interesting to probe the sense of gas rotation beyond the SAURON field of view, up to the optical border of the stellar disk - it is a sure chance to estimate the space scale of external gas accretion. By measuring oxygen abundance in the extended gaseous disk, we can also make a choice between various possible accretion sources. And the last, but not the least aim of our analysis is a search for the secondary stellar component which may be coupled with the counterrotating gas: the detailed star formation history in the counterrotating gaseous disk is probably coupled with the accretion history and so allows to reconstruct the accretion history and to identify its source. We have fulfilled long-slit spectral observations of IC 719 and are presenting now their 
TABLE 1

Global parameters of the galaxies

\begin{tabular}{lc}
\hline Galaxy & IC 719 \\
Type $\left(\mathrm{NED}^{1}\right)$ & $\mathrm{S} 0 ?$ \\
$R_{25}, \mathrm{kpc}\left(\mathrm{NED}_{\mathrm{RC}} \mathrm{RC}^{2}\right)$ & 5.5 \\
$B_{T}^{0}\left(\mathrm{LEDA}^{3}\right)$ & 13.66 \\
$M_{B}$ (LEDA) & -18.6 \\
$M_{K}$ (ATLAS-3D) & -22.7 \\
$V_{r}(\mathrm{NED})$ & $1860 \mathrm{~km} \cdot \mathrm{s}^{-1}$ \\
Distance, Mpc (ATLAS-3D) & 29.4 \\
Inclination (LEDA) & $90^{\circ}$ \\
$P A_{\text {phot }}(\mathrm{LEDA})$ & $52^{\circ}$ \\
$V_{\text {rot }} \mathrm{sin} i, \mathrm{~km} \cdot \mathrm{s}{ }^{-1},(\mathrm{LEDA}, \mathrm{HI})$ & $114.4 \pm 6.6$ \\
$\sigma_{*}, \mathrm{~km} \cdot \mathrm{s}^{-1},(\mathrm{LEDA})$ & 121 \\
$M_{H I}, 10^{9} M_{\odot}^{4}$ & 0.54 \\
$M_{H_{2}}, 10^{8} M_{\odot}^{5}$ & 1.8 \\
\hline${ }^{1} \mathrm{NASA} / \mathrm{IPAC}$ Extragalactic Database \\
${ }^{2}$ Third Reference Catalogue of Bright Galaxies \\
${ }^{3}$ Lyon-Meudon Extragalactic Database \\
${ }^{4}$ Grossi et al. (2009); Serra et al. \\
${ }^{5}$ Young et al. (2011)
\end{tabular}

results. Also we involve panoramic spectroscopic data from the ATLAS-3D survey (Cappellari et al. 2011) into our analysis. The layout of the paper is the following: Section 2 describes our observations and data reduction, Section 3 is dedicated to description of our analysis approaches, in Section 4 we discuss the results on kinematics of the stellar and gaseous components and their chemical compositions. In Section 5 we conclude.

\section{OBSERVATIONS AND DATA REDUCTION}

We used two datasets obtained from long-slit and integral-field spectroscopy. The long-slit spectroscopic observations were made with a new universal spectrograph SCORPIO-2 (Afanasiev \& Moiseev 2011) at the prime focus of the Russian 6-m BTA telescope operated by the Special Astrophysical Observatory, Russian Academy of Sciences. IC 719 was observed in November 2011 with the $1^{\prime \prime}$ slit aligned along the major axis, with the total exposure times of $7 \times 1200 \mathrm{sec}$. The median atmosphere seeing FWHM during these observations was 1.5 arcsec. We used the VPHG1200 grizm providing an intermediate spectral resolution $\mathrm{FWHM} \approx 3.5 \AA$ in a wavelength region from 4300 to $7300 \AA$. This spectral range included a set of strong absorption and emission features making it suitable to study both internal stellar and gaseous kinematics and the stellar populations of the galaxy. The slit was $6^{\prime}$ in length providing a possibility to use the edge spectra to evaluate the sky background. The CCD chip E2V CCD42-90, with a format of $2048 \times 4600$, using in the $1 \times 2$ binning mode provided spatial scale of 0.357 " / px and a spectral sampling of $0.84 \AA / \mathrm{px}$.

The preliminary data reduction was identical to that applied to the lenticular galaxy NGC 7743 described in Katkov et al. (2011a). Briefly, the primary data reduction comprised bias subtraction, flat-fielding, cosmic ray hit removal, building the wavelength solution using the $\mathrm{He}-\mathrm{Ne}-\mathrm{Ar}$ arc-line spectra. To subtract the sky background, we invented a rather sophisticated approach. We constructed the spectral line spread function (LSF) model varied along and across the wavelength direction by using the twilight spectrum (Katkov \& Chilingarian 2011b). The final stages of the long-slit spectra reduction were night sky spectrum subtraction taking into account the LSF variations, linearization and accounting spectral sensitivity variation using the spectrum of a spectrophotometrical standard star. The error frames were computed using the photon statistics and processed through the same reduction steps as the data.

We used also the data obtained with the integral-field spectrograph SAURON (Bacon et al. 2001) mounted at the $4.2 \mathrm{~m}$ William Hershel Telescope, La Palma, in the frame of the ATLAS-3D survey (Cappellari et al. 2011). The raw science and calibration exposures were retrieved from the open Isaac Newton Group Archive of the Cambridge Astronomical Data Center. The field of view of the spectrograph is $44 \times 38$ spatial elements (spaxels) at a $0.94^{\prime \prime}$ scale per spaxel. The SAURON spectral range is $4800-5350 \AA$ with spectral resolution FWHM of $\approx 4 \AA$. For details of how we reduced the SAURON raw data see Sil'chenko \& Chilingarian (2011).

\section{INTERNAL KINEMATICS AND STELLAR POPULATIONS}

\subsection{NBURSTS fitting}

We derived the parameters of internal stellar kinematics and stellar population properties of IC 719 by fitting high-resolution PEGASE.HR (Le Borgne et al. 2004) single (simple) stellar population (SSP) models, which were computed with the Salpeter stellar initial mass function, to our spectra by using the NBURSTS full spectral fitting technique (Chilingarian et al. 2007a,b). Before the main minimization loop a grid of the SSP spectra with a fixed set of ages and metallicities is convolved with the instrumental response of the spectrograph (LSF). The non-linear least-square fitting algorithm is applied to select a template spectrum from the grid of the SSP models by specifying its luminosityweighted ('SSP-equivalent') age $t$ and metallicity $[\mathrm{Z} / \mathrm{H}]$. Then we convolve the template spectrum with a lineof-sight velocity distribution (LOSVD) approximated by the Gauss-Hermite parametrization up to the $4^{\text {th }}$ moment, i.e. $v, \sigma, h_{3}$ and $h_{4}$ (van der Marel \& Franx 1993). Multiplicative Legendre polynomials are also in-
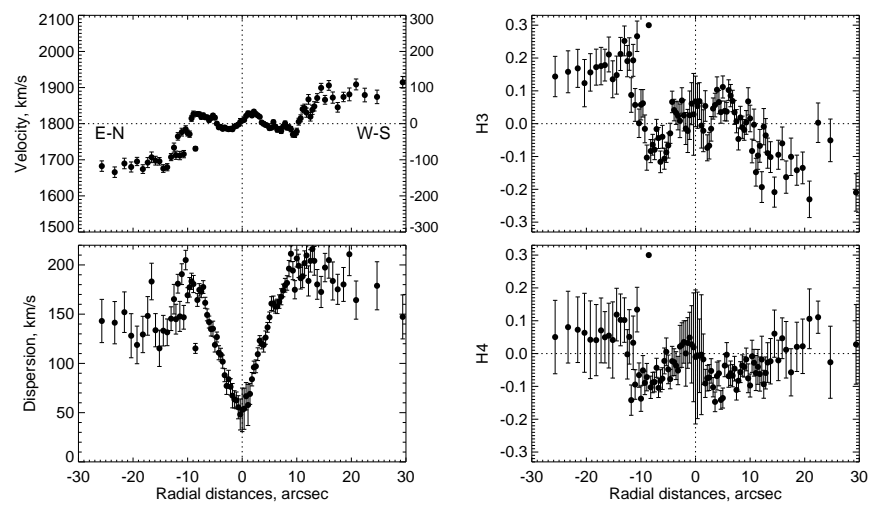

FIG. 2.- The results of the analysis of the long-slit spectrum for IC 719: left top - stellar LOS velocity profile; left bottom stellar velocity dispersion profile, right top and bottom - Hermite coefficients $h_{3}, h_{4}$. The binning is made along the slit to maintain $S / N=15$ per pixel. 

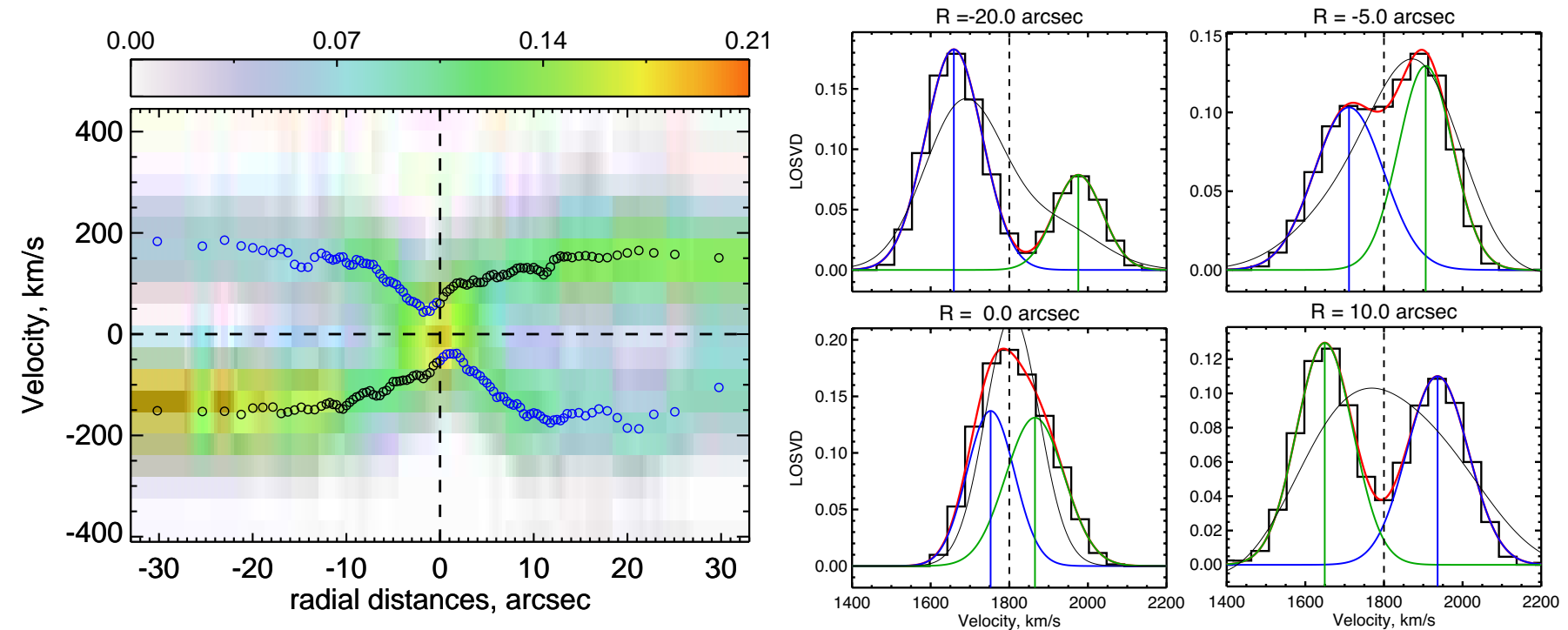

FIG. 3.- The view of the stellar LOSVD for IC 719 obtained by non-parametric technique. Left - stellar position-velocity diagram for the long-slit spectrum. Black and blue circles mark position of gaussian centres for two-gaussian decomposition. Right - cross-sections of the PV diagram at the different radial distances $(r=-20,-5,0,10$ arcsec $)$. Solid black line presents recovered stellar LOS at given distances, red line - model of the sum of two separate gaussians (green and blue lines). Thin black lines correspond to Gauss-Hermite approximation of the stellar LOS recovered under one-component approach using the NBURSTS fitting technique (see Section 3.1).

cluded to take into account possible internal dust reddening and residual spectrum slope variations due to the errors in assumed instrument spectral response. Ionizedgas emission lines and traces of the subtracted strong airglow lines do not affect the solution due to masking of the narrow 20- $\AA$-wide regions around them. Moreover, excluding age-sensitive Balmer lines from the fit neither biases age estimates nor degrades significantly the quality of the age determination (see details in Chilingarian (2009) and Appendix A2 in Chilingarian et al. (2007a)). In order to achieve the required signal-to-noise ratio per spatial bin we performed adaptive binning of the long-slit spectra as well as of the integral-field spectra by using Voronoi-tessellation scheme (Cappellari \& Copin 2003). Fig. 2 shows the parameters of the stellar kinematics for IC 719 derived in such a way.

The uncertainties of the parameters derived were returned by the minimization procedure using flux errors propagation through all data reduction steps. Chilingarian et al. (2007a) performed some Monte Carlo simulations for the NBURSTS fitting technique and demonstrated the consistency between the uncertainties of the parameters returned by the minimization procedure and the real error distributions.

By applying this approach, we found that the stellar velocity dispersion demonstrates two off-centred maxima and high absolute values of $h_{3}, h_{4}$, up to 2.5 (see Fig. 2). Such values of $h_{3}, h_{4}$ correspond to a strongly asymmetric stellar LOSVD. In order to analyze in detail the stellar LOSVD we probed then a non-parametric recovery technique, which does not require a priori knowledge of the LOSVD shape.

\subsection{Non-parametric LOSVD}

The only assumption we used is that an observed galaxy spectrum logarithmically rebinned in the wavelength domain can be represented by convolution of a velocity distribution $\mathcal{L}$ and a typical stellar (template) spectrum. We used the SSP model from the NBURSTS fitting, preconvolved with the LSF output, as a template spectrum. The convolution can be considered as a linear inverse problem whose solution (vector $\mathcal{L}$ ) can be estimated by the least squares method. The solution is very sensitive to the noise in the data. Hence we chose to regularize the problem be requiring the LOSVD to be smooth. In order to do so, we choose the smoothing regularizing matrix operator in the following form: $\mathcal{P}(\mathcal{L})=\mathcal{L}^{T} \cdot \mathcal{D}_{i}^{T} \cdot \mathcal{D}_{i} \cdot \mathcal{L}$, where matrix $\mathcal{D}_{i}$ - is the $i^{\text {th }}$ order difference operator. For details, see chapter 19 of "Numerical Recipes" (Press et al. 2007). The regularized inverse problem, where the function to be minimized is given by $\Omega=\chi^{2}+\lambda \mathcal{P}(\mathcal{L})$, can be expressed as a linear matrix equation and solved by the BVLS algorithm (Lawson \& Hanson (2004)) which allows to constrain the solution by positive values. We performed numerous tests by varying the order of the difference operator and penalization coefficient $\lambda$, and concluded that the optimal values are $i=5$ and $\lambda=0.01 \lambda_{e}$. The choice of $\lambda$ equal to $\lambda_{e}$ will tend to make both parts of the minimization function $\Omega$ to have comparable weights. The technique used here is similar to that developped by Kuijken \& Merrifield (1993). The stellar LOSVD derived from the long-slit data for IC 719 is presented in Fig. 3 as a position-velocity diagram. The GaussHermite representation of the stellar LOS recovered under one-component approach also is shown at Fig. 3. One can see that there is no acceptable agreement between the non-parametric approach and the Gauss-Hermite representation. Therefore, the Gauss-Hermite parametrization cannot be applied to the complex LOSVD where two comparable peaks have a significant velocity separation.

\subsection{Two-component fitting}

Fig. 3 demonstrates that the stellar LOSVD for IC 719 has a complex two-peaked structure. We have decomposed the stellar LOSVD derived by the non-parametric 

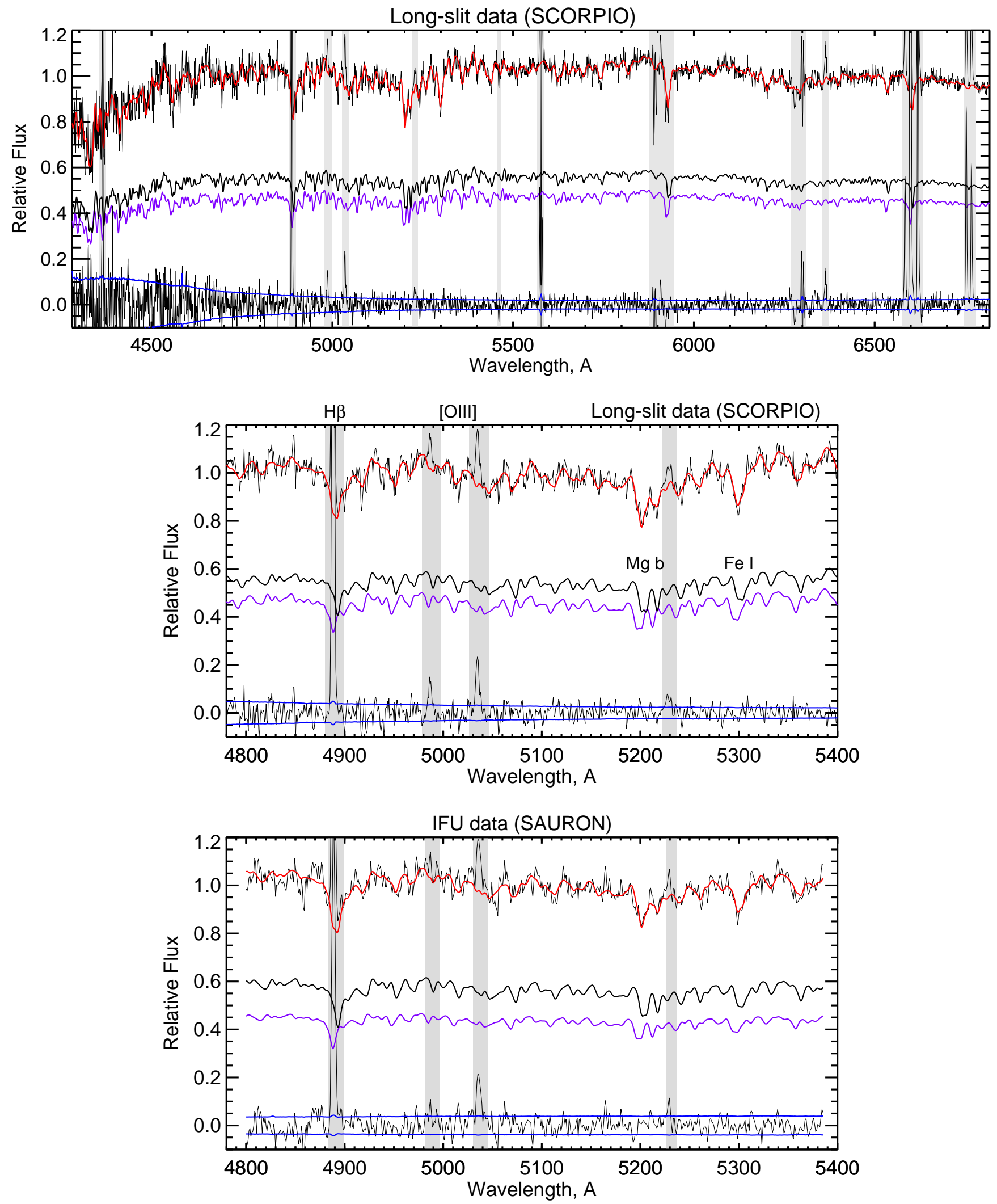

FIG. 4.- The galaxy spectrum in the spatial bin at 7-8 arcsec south-west from the galaxy centre extracted from the long-slit (top and middle panels) and IFU dataset (bottom panel). The data are shown in black, the red line shows best-fit model which is the sum of the two stellar components (purple and black). Residuals and noise level are also shown. The grey vertical stripes correspond to masked narrow regions around emission lines which are excluded from fitting procedure. 
technique into two separate gaussians in order to evaluate line-of-sight velocity and velocity dispersion of every component. The centers of the gaussians are overplotted at the position-velocity diagram as black and blue circles (Fig. 3).

In order to study the stellar populations of the counterrotating disks we separated their contributions into the observed spectrum by using an advanced implementation of the NBURSTS full spectral fitting technique. In general, this approach is similar to that described in section 3.1 but now we use a two-component model where the optimal template consists of a linear combination of two SSPs characterized by their ages and metallicities, each of them convolved with its own Gaussian-shaped LOSVD. The relative luminosity contribution of one of the components, $p$, is included into the parameter set of the minimization loop. The contribution of the other component is equal to $1-p$. The multiplicative polynomial continuum factor is similar for both components. By undertaking the re-analysis of the long-slit data, we have decided to keep the velocity dispersions equal to the values, which were derived from the decomposition of the non-parametric LOSVD into two Gaussians. The range of the stellar velocity dispersions obtained for the both Gaussian components is rather narrow, $\sigma_{\star}=60-80$ $\mathrm{km} / \mathrm{s}$, so during the IFU data analysis we fixed velocity dispersions of the components to be equal to $70 \mathrm{~km} / \mathrm{s}$. It is why there is no error bars at the velocity dispersion profiles in Fig. 5 and there are no velocity dispersion maps in Fig. 6. If we take the dispersions of both components as free parameters, the velocity profiles and profiles of the stellar population properties look more noisy due to the additional degree of freedom.

In Fig. 4 we demonstrate the quality of our spectral fitting results by plotting the observed and model spectra in the same, arbitrarily taken, spatial bin for two datasets - our long-slit data and the SAURON IFU data. The kinematical and stellar population parameters of the stellar components separated in such a way as well as the relative luminosity contributions and light profile decomposition results are shown in Fig. 5 for the long-slit data and in Fig. 6 for the SAURON data. We have succeeded to extract two stellar components counterrotating with the similar velocities about $\sim 200 \cdot \sin i$ $\mathrm{km} / \mathrm{s}$. The component which corotates the ionized gas (see below) gives lower contribution to the disk surface brightness at $R>10^{\prime \prime}$ than its counterpart, so we would treat is as a 'secondary' stellar component; in general it looks somewhat younger $\left(T_{\text {sec }} \approx 2.5 \mathrm{Gyr}\right)$ and more metal rich $\left([\mathrm{Z} / \mathrm{H}]_{\text {sec }} \approx-0.1 \div 0.0\right.$ dex $)$ than the other one $\left(T_{\text {main }} \approx 4 \mathrm{Gyr},[\mathrm{Z} / \mathrm{H}]_{\text {main }} \approx-0.3 \div-0.2 \mathrm{dex}\right)$.

Two derived counterrotating stellar components seem to be dynamically cold disk structures, with their ratio of the maximum rotation velocity to velocity dispersion $V_{\text {rot }} / \sigma \approx 3$ for each component. The identification of the large-scale stellar structures in IC 719 as the cold flat disks is supported by surface photometry. We extracted azimuthally averaged surface brightness profiles in the SDSS $g, r, i$-bands and in the UKIDSS $K$-band using the IRAF task ELLIPSE (Jedrzejewski 1987) (see Fig. 1) and found that the profiles are quasi exponential with a compact nuclear concentration and no presence of a massive bulge. At the radii of $r=7^{\prime \prime}-10^{\prime \prime}$ one can see an excess of the surface brightness, which apparently corresponds to the concentration of the secondary stellar component. This luminosity excess looks like a ring structure at the bivariate picture of the residuals between $K$-band image and exponential disk model constructed over the outer regions $\left(r>20^{\prime \prime}\right)$ of the galaxy by using the GALFIT package (Peng et al. 2002).

\subsection{Ionized gas}

An emission-line spectrum of every spatial bin was obtained by subtracting the stellar contribution (i.e., the best-fitting stellar population model) from the observed spectrum. This step provided a pure emission spectrum uncontaminated by absorption lines of the stellar component that is especially important for the Balmer lines. Then we fitted emission lines with Gaussians preconvolved with the instrumental LSF in order to determine the line-of-sight (LOS) velocities of the ionized gas and emission-line fluxes. The LOS velocity profiles and maps of velocities and fluxes of the emission lines are shown in Fig. 5 and Fig. 7, respectively.

The line fluxes have been corrected for the internal interstellar extinction as well as for the Galactic extinction according to Schlegel et al. (1999). The color excess $\mathrm{E}(\mathrm{B}-\mathrm{V})$ corresponding to the internal dust reddening was determined from Balmer decrement using the theoretical line ratios $F(H \alpha) / F(H \beta)=2.87$ for the electron temperature $T_{e}=10000 \mathrm{~K}$ (Osterbrock \& Ferland 2006) and the parametrized extinction curve (Fitzpatrick 1999).

We plotted our measurements of emission lines onto the classical excitation-type diagnostic diagrams to identify the gas ionization source (Fig. 8). One can see that all measurements are located in the area corresponding to photoionization by hot stars except a few central points which correspond to shock-like excitation or to that powered by a weak (LINER) AGN.

Due to the wide spectral range of the SCORPIO longslit spectra covering a set of strong emission lines $(\mathrm{H} \beta$, $[\mathrm{O}$ III $] \lambda 5007, \mathrm{H} \beta,[\mathrm{N} \mathrm{II}] \lambda 6548, \lambda 6583,[\mathrm{~S} \mathrm{II}] \lambda 6717, \lambda 6730)$ we are able to determine oxygen and nitrogen abundances of the ionized gas. To do this we used so-called NS-calibration method (Pilyugin \& Mattsson 2011) which does not require [O II] $\lambda 3727+\lambda 3729$ lines. This NS calibration uses strong emission lines $\mathrm{O}^{++}, \mathrm{N}^{+}$, $\mathrm{S}^{+}$and is based on the spectra of $\mathrm{H}$ II regions with measured electron temperatures as a calibration data set. In order to compare oxygen and nitrogen abundances of the ionized gas with the metallicity of the stellar component, we plot all the radial profiles of the abundances in Fig. 9.

\section{DISCUSSION}

The LOSVD of IC 719 can be easily splitted by eye into two counterrotating stellar components (Fig. 3). So for this galaxy we have undertaken the spectrum fitting by two SSPs with different kinematical and stellar population parameters.

The early analysis by the ATLAS-3D team that was using the kinemetry approach involving one-component stellar population with the unified kinematics, returned the classification of IC 719 as a rotator, slow in the inner part. The galaxy was also included into a so called ' $2 \sigma$ ' type, so was treated as a galaxy having two off-centered maxima of the stellar velocity dispersion 

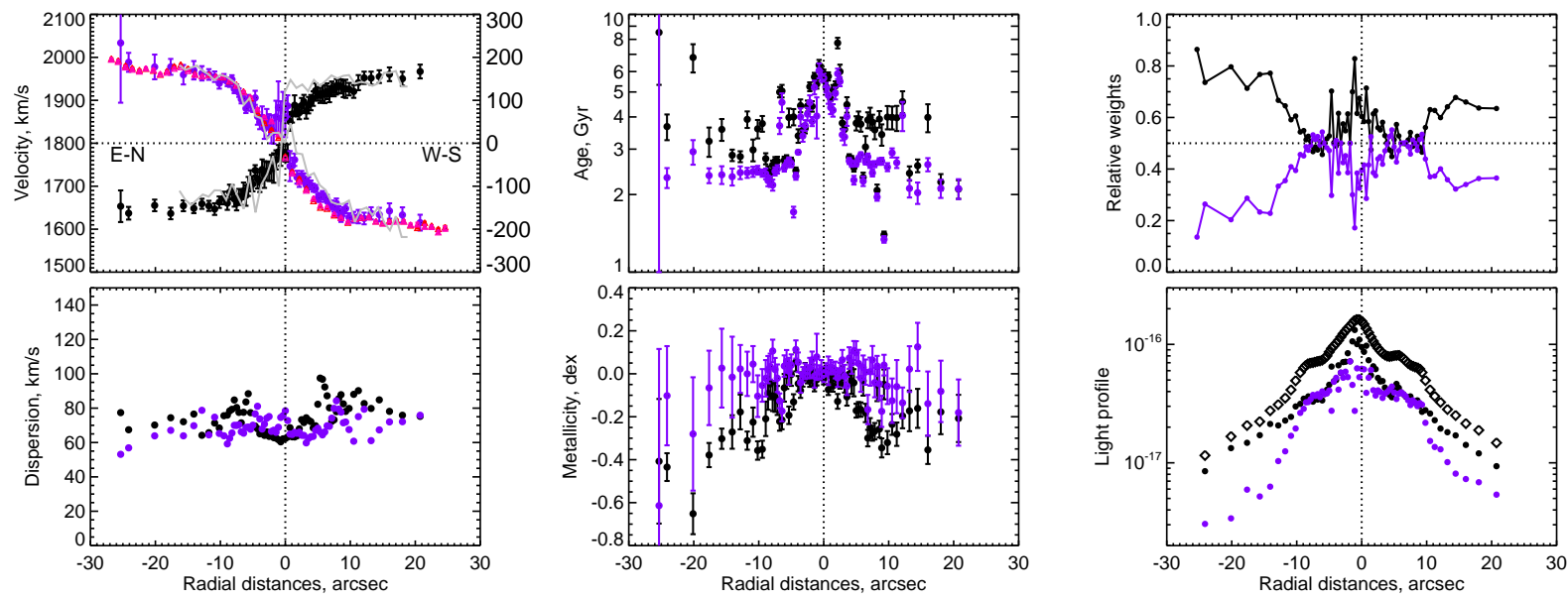

FIG. 5. - The results of the two-component analysis of the long-slit spectrum for IC 719: left top - LOS velocity profiles, both for the stars (black and blue dots) and for the ionized gas (pink triangles for the [N II] $\lambda 6583$ ); left bottom - stellar velocity dispersion profiles, mid top - SSP-equivalent age profiles for two stellar components, mid bottom - metallicity profiles for two stellar components; right top - relative contribution of components, right bottom - open diamonds shows light profile which was obtained by integration spectra along long-slit, close symbols correspond to light profiles for each component. The binning is made along the slit to maintain $S / N=20$ per pixel. The grey lines at left panel correspond to SAURON data extracted along major axis.
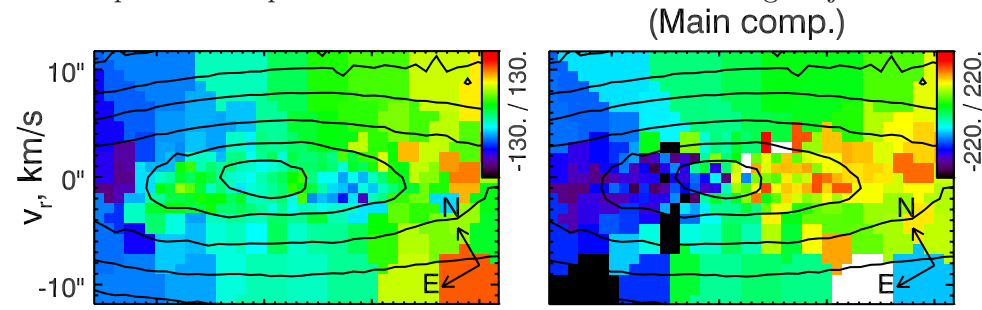

(Secondary comp.)
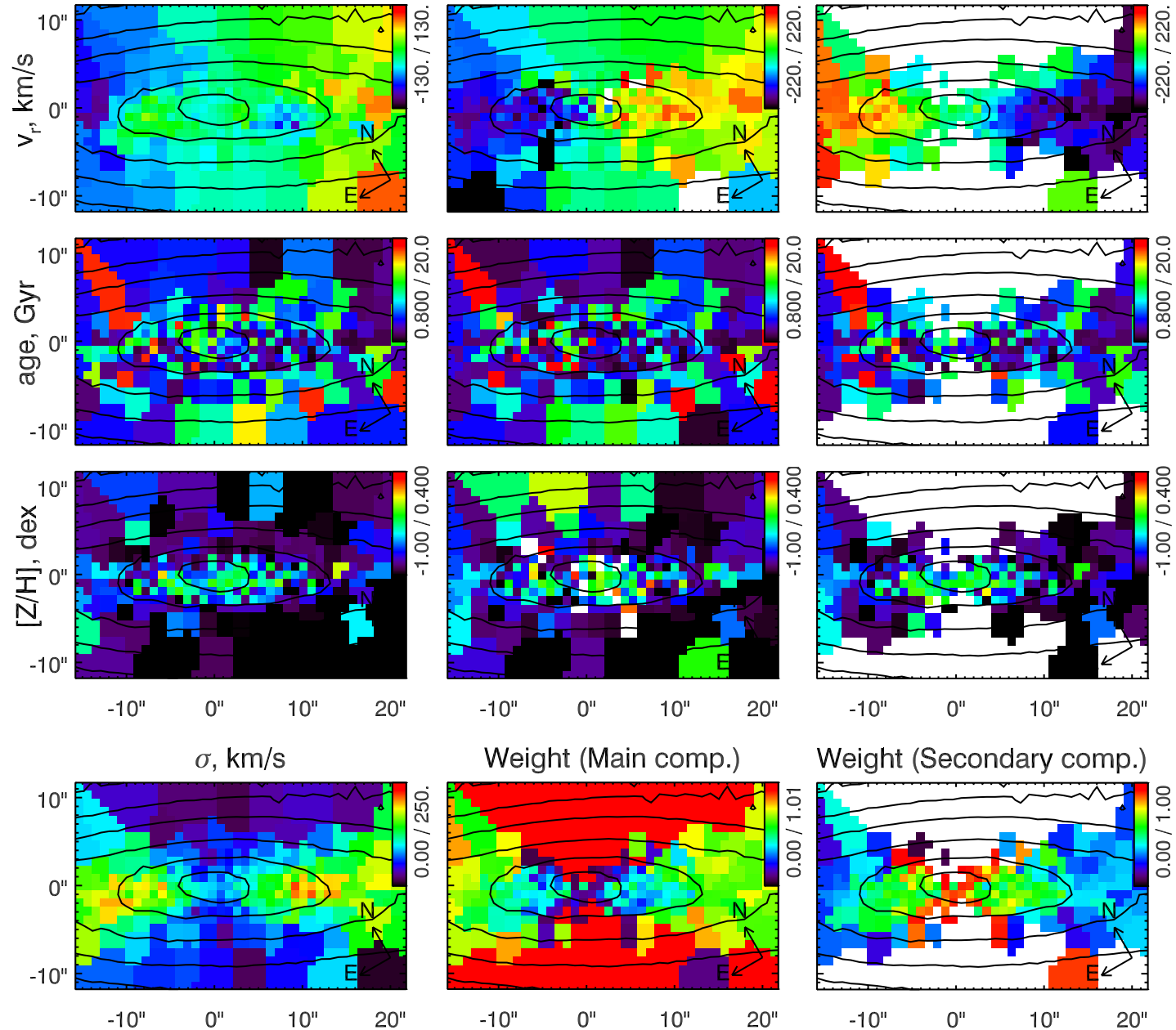

Weight (Secondary comp.)

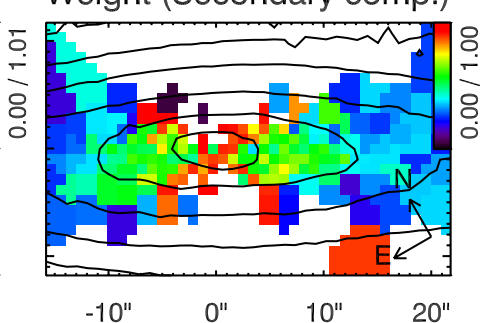

FiG. 6. - The results of the analysis of the SAURON spectra for IC 719: left column shows the results of fitting by a single stellar component, and the central and right columns show the two-component fitting results. From top to bottom the LOS velocity field, the SSP-equivalent age map, the metallicity map are presented. The bottom row consists of stellar velocity dispersion field for one-component stellar fitting (left column) and the maps of relative contribution of every stellar components (mid and right columns). Note that color bar has a different range for the velocity fields at left column and other columns. 
(Krajnovic et al. 2011; Emsellem et al. 2011). In fact, when the correct approach including two kinematically (and evolutionarily) decoupled SSPs is applied, we find two fast (counter-) rotating stellar components even in the innermost region (Fig. 6). The off-centered spots of visibly high stellar velocity dispersion (or of a widened LOSVD) are in fact the locations the nearest to the center where two LOSVD branches start to separate. The gaseous component extracted from the SAURON data after the fitting by two SSP rotates just as one of the stellar components; the surface intensity of the $\mathrm{H} \beta$ emission demonstrates a ring-like distribution with the radius of about $10^{\prime \prime}$ (Fig. 7).

By applying our two-component fitting to the IC 719 long-slit data obtained with the SCORPIO (Fig. 5), we have obtained two counterrotating stellar components once again; one of the LOS stellar velocity profiles coincides perfectly with the gas velocity profile, the other is mirroring. The amplitudes of both stellar LOS velocity profiles are similar. Beyond the central part of the galaxy, the stars corotating the ionized gas look somewhat younger $\left(T_{\text {sec }} \approx 2.5 \mathrm{Gyr}\right)$ and more metal-rich $\left([\mathrm{Z} / \mathrm{H}]_{\text {sec }} \approx-0.1 \div 0.0 \mathrm{dex}\right)$ than the counterrotating component $\left(T_{\text {main }} \approx 4 \mathrm{Gyr},[\mathrm{Z} / \mathrm{H}]_{\text {main }} \approx-0.3 \div-0.2\right.$ dex). The BPT diagram diagnostics of the pure emissionline spectrum, which was obtained by subtracting the model stellar population spectrum from the observed one, has shown (Fig. 8) the following picture: the gas excitation in the nucleus is shock-like or powered by a weak (LINER) AGN, but in the ring and beyond it the gas is ionized by young stars. After measuring the gas abundances by using the emission line flux ratios and NS calibration relation from Pilyugin \& Mattsson (2011), we find that in general the ionized gas metallicity is higher then the metallicity of the main stellar component and tends to be smaller or equal to the metallicity of the secondary component (see Fig. 9).

For the first time the technique of spectroscopic decomposition was presented by Coccato et al. (2011). They simultaneously measured both the kinematics and stellar population properties of the counter-rotating stellar disks of NGC 5719 and found that the stellar component, which rotates in the same direction as the ionized gas, is younger, less rich in metals, more $\alpha$-enhanced, and less luminous than the main galaxy disk. Their investigation defiantly confirmed the gas accretion scenario for the secondary stellar disk of NGC 5719. Later this team studied stellar populations in the two more well-known galaxies NGC 3593 and NGC 4550 hosting counter-rotating disks (Coccato et al. 2013). In the both galaxies the secondary stellar components are less massive, more metal poor and $\alpha$-enhanced than the main galaxy stellar disks, and co-rotate the ionized gas. These findings rule out an internal origin of the secondary stellar component and favor a gas-accretion scenario. NGC 4550 was also studied by Johnston et al. (2013). They obtained that the age of the secondary disk is a bit younger than it was evaluated by Coccato et al. (2013). Both papers concluded that the most likely formation mechanism of the secondary stellar component in NGC 4550 is unusual gas accretion history.

What can be the origin of the counterrotating gas in IC 719? A standard answer could be 'merging' or 'interaction'. The chemistry of the gas which is not very metal-poor (at least within the stellar body of the galaxy) excludes the version that the extended counterrotating gaseous disk consists of the relic baryons never processed through the stars, so there is no reason to suspect cold accretion from large-scale cosmological filaments. Meantime, IC 719 constitutes an isolated non-interacting pair with IC 718 - a late-type galaxy of similar luminosity. Moreover, neutral hydrogen mapping reveals huge gas cloud embedding both galaxies (Grossi et al. 2009; Serra et al. 2012). The HI extended disk of IC 719 looks coplanar to the stellar disk of IC 719 though is much more extended - up to $100 \mathrm{kpc}$ from the galactic center. Is it an interaction with gas flows directed from the latetype neighbour onto the early-type one? Merging with some third galaxy seems improbable: the high mass of the gas (at least 0.7 billion solar masses), together with the modest stellar mass of the galaxy, $6 \cdot 10^{10}$ solar masses (see $M_{K}$ in the Table 1), implies the necessity of merging galaxies with the mass ratio larger than 1:10; it means that the thin stellar disk could not survive during such merging (Walker et al. 1996; Thakar \& Ryden 1996) unless very specific conditions were provided - intense hot halo gas cooling after the merger (Moster et al. 2012) (meantime, X-ray haloes are not reported around these galaxies) or strictly coplanar satellite orbiting before the merger. Meantime both stellar disks of IC 719 are rather cold dynamically.

However, it is evident that the inner ionized-gas component having the same angular momentum has a strong connection with the secondary stellar disk, though star formation in the disk have started perhaps recently, because the bulk stellar populations in the large-scale stellar disk of the galaxy are of intermediate age, $>2$ Gyr. One can consider two accretion scenarios of the gas onto the galaxy. In the frame of the first one, the gas was acquired through a single accretion event but then two starbursts have happened. Early starburst has given rise to formation of the secondary stellar component and the last one continues now. The second scenario consists of two accretion events, each with a subsequent starburst. We prefer the latter scenario of gas accretion because the former one is inconsistent with the gas metallicity measured by us. Indeed, under the first scenario we expect that the metallicity of the self-enriched gas would be higher then the stellar metallicity of the secondary component but the Fig. 9 shows the opposite relation. The second scenario allows that the metallicity of the mixture of the metal-poor external gas with the inner enriched gaseous fraction may be below the metallicity of the secondary stellar component, that is consistent with our measurements.

\section{CONCLUSIONS}

By adding our long-slit spectral observations to the panoramic spectroscopy with the SAURON, we have detected extended counterrotating gaseous disk as well as a secondary stellar component corotating the ionized gas in the lenticular galaxy IC 719. The gas counterrotation can be traced up to the optical borders of the galaxies; moreover, the ionized-gas velocity profiles obtained by us are more extended than the stellar velocity profiles extracted from the same spectral data. The gas emission-line surface intensity demonstrates ring-like distributions within the disk of the galaxy; according to the BPT-diagnostics 

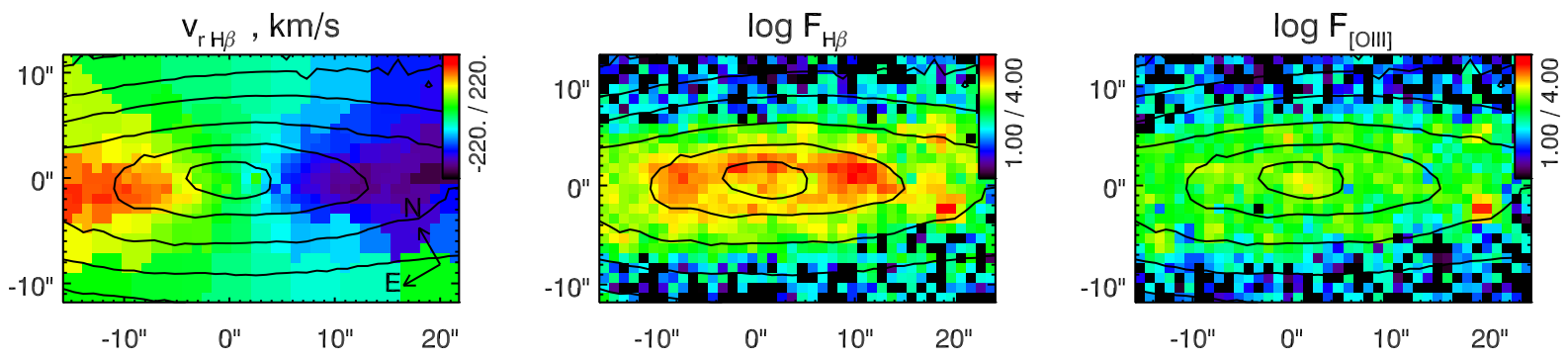

FIG. 7. - The results of the emission-line component analysis by using the SAURON data: left - the ionized gas velocity field constructed using $\mathrm{H} \beta$ line, mid - the intensity map for the emission line $\mathrm{H} \beta$, right - the intensity map for the emission line [O III] $\lambda 5007$
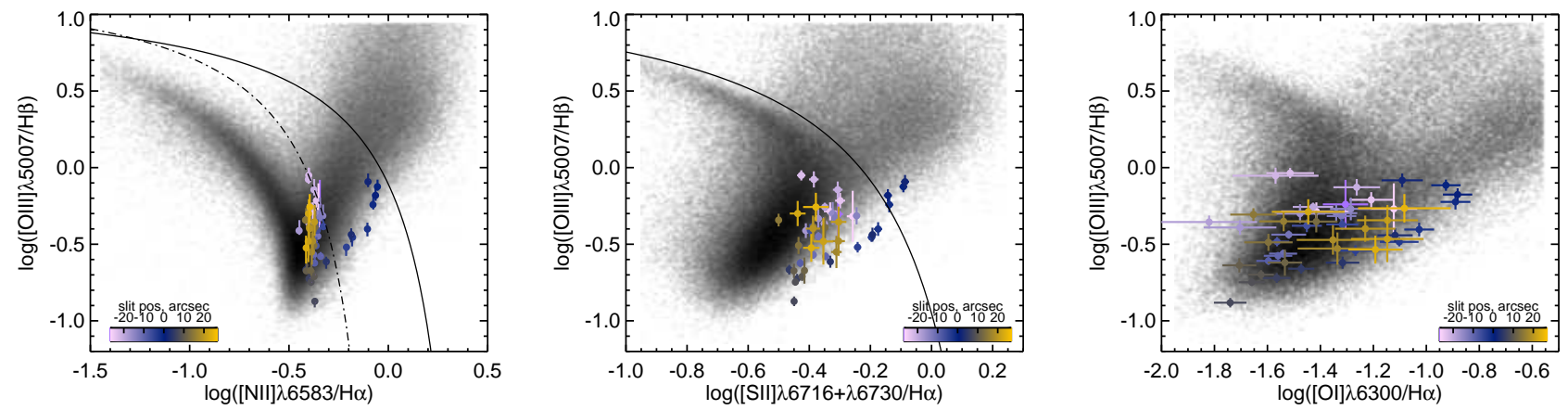

FIG. 8. - Excitation diagnostic diagrams comparing the emission-line ratios: $[\mathrm{N} \mathrm{II}] / \mathrm{H} \alpha$ vs. [O III] $/ \mathrm{H} \beta$ (left), [S II] $/ \mathrm{H} \alpha$ vs. [O III] $/ \mathrm{H} \beta$ (mid), $[\mathrm{OI}] / \mathrm{H} \alpha$ vs. $[\mathrm{O} \mathrm{III}] / \mathrm{H} \beta$ (right). The points show the extracted emission line ratios from the long-slit spectra for IC 719 . The color of points corresponds to the distance from the galaxy center. Distribution of measurements of the line ratios for galaxies from SDSS survey with high signal-to-noise ratio $(S / N>3$ in every line) is showed by grey color. The black curves, which separate the areas with the AGN/LINER excitations from areas with the star-formation-induced excitation, are taken from Kauffmann et al. (2003) (dash-dot curve) and from Kewley et al. (2006) (solid curve).

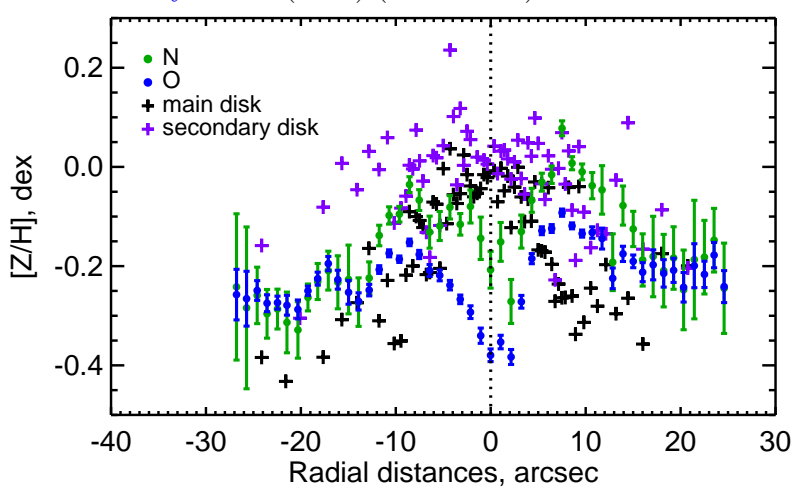

FIG. 9. - Radial profiles of gaseous (filled circles) and stellar abundances (crosses). Green and blue circles with error bars correspond to nitrogen and oxygen abundances with subtracting solar value, respectively. We adopted a solar nitrogen abundance $12+\log N / H$ to be 7.83 then the solar oxygen abundance $12+\log O / H$ is 8.69 (Asplund et al. 2009). The abundances in the nucleus are unreliable due to different gas excitation mechanism there.

from the line ratio confrontation, the gas in the rings is excited by young stars, so the extended disk of the IC 719 possess rather intense current star formation. We have demonstrated that the accretion history of the external gas onto IC 719 consist of two events, each with a subsequent starburst.

In any case, this field lenticular galaxy is growing its large-scale counterrotating stellar disk just now promising to become in the nearest future an analog of NGC 4550 - the S0 galaxy which has two equal-mass counterrotating stellar disks (Rubin et al. 1992; Rix et al. 1992) and no current star formation (Crocker et al. 2011).

This research is partly based on data obtained from the Isaac Newton Group Archive which is maintained as part of the CASU Astronomical Data Centre at the Institute of Astronomy, Cambridge. We have made use of the NASA/IPAC Extragalactic Database (NED) which is operated by the Jet Propulsion Laboratory, California Institute of Technology, under contract with the National Aeronautics and Space Administration. In this study, we used the UKIDSS DR8 image data available through the WFCAM science archive and SDSS DR9 data. Funding for the SDSS and SDSS-II has been provided by the Alfred P. Sloan Foundation, the Participating Institutions, the National Science Foundation, the U.S. Department of Energy, the National Aeronautics and Space Administration, the Japanese Monbukagakusho, the Max Planck Society, and the Higher Education Funding Council for England. The SDSS Web Site is http://www.sdss.org/. We wish to thank the anonymous referee for his/her useful comments and suggestions. We acknowledge the usage of the HyperLeda database. This work was supported by the Ministry of Education and Science of the Russian Federation and Russian Foundation for Basic Research (projects no. 10-02-00062a, no. 12-02-00685) and Presidential Grant no. MD-3288.2012.2. IYK is also grateful to Dmitry Zimin's non-profit Dynasty Foundation.

\section{REFERENCES}


Asplund, M., Grevesse, N., Jacques Sauval, A., Scott, P. 2009, ARA\&A, 47, 481

Bacon, R., Copin, Y., Monnet, G., et al. 2001, MNRAS, 326, 23

Baldwin, J. A., Phillips, M. M., Terlevich, R. 1981, PASP, 93, 5

Bertola, F., Buson, L. M., Zeilinger, W. W., 1992, ApJ, 401, L79

Bertola, F., Cinzano, P., Corsini, E. M., et al. 1996, ApJ, 458, L67

Cappellari, M., Emsellem, E., Krajnovic, D., et al. 2011, MNRAS, 413,813

Cappellari, M. \& Copin, Y. 2003, MNRAS, 342, 345

Chamaraux, P., Balkowski, C., Fontanelli, P. 1987, A\&AS, 69, 263

Chilingarian I. V., Prugniel P., Sil'chenko O. K., Afanasiev V. L. 2007a, MNRAS, 376, 1033

Chilingarian I. V., Prugniel P., Sil'chenko O. K., Koleva M. 2007b, in Stellar Populations as Building Blocks of Galaxies, (IAU Symp. 241), eds. A. Vazdekis \& R. F. Peletier, p. 175, arXiv:0709.3047

Chilingarian I. V. 2009, MNRAS, 394, 1229

Chung, A., Koribalski, B., Bureau, M., van Gorkom, J. H. 2006 MNRAS, 370,1565

Ciri, R., Bettoni, D., Galletta, G. 1995, Nature, 375, 661

Coccato, L., Morelli, L., Corsini, E. M., et al. 2011, MNRAS, 412 L113

Coccato, L., Morelli, L., Pizzella, A., et al. 2013, A\&A, 549, A3

Crocker, A. F., Bureau, M., Young, L. M., Combes, F. 2011, MNRAS, 410, 1197

Davis, T. A., Alatalo, K., Sarzi, et al. 2011, MNRAS, 417, 882

Emsellem, E., Cappellari, M., Krajnovic, D., et al. 2011, MNRAS, 414, 888

Fitzpatrick, E. E. 1999, PASP, 111, 63

Garcia-Burillo, S., Sempere, M. J., Bettoni, D. 1998, ApJ, 502, 235

Grossi, M., di Serego Alighieri, S., Giovanardi, et al. 2009, A\& A, 498, 407

Haynes, M. P., Jore, K. P., Barrett, E. A., Broeils, A. H., Murray, B. M. 2000, AJ, 120, 703

Jedrzejewski, R. I. 1987, MNRAS, 226, 747

Johnston, E. J., Merrifield, M. R., Aragón-Salamanca, A., Cappellari, M. 2013, MNRAS, 428, 1296

Katkov, I. Yu., Moiseev A. V., Sil'chenko O. K. 2011, ApJ, 740, 83

Katkov, I. Yu., \& Chilingarian I. V. 2011, in ASP Conf. Ser. 442, Astronomical Data Analysis Software and Systems XX, ed. I.N.Evans et al. (San Francisco, CA: ASP), 143

Katkov, I. Yu., Chilingarian, I. V., Sil'chenko, O. K., Zasov, A. V., Afanasiev, V. L. 2011, Balt. Astron., 20, 453
Kauffmann, G., Heckman, T. M., Tremonti C., et al. 2003, MNRAS, 346, 1055

Kewley, L. J., Groves, B., Kauffmann, G., Heckman, T. 2006, MNRAS, 372, 961

Krajnovic, D., Emsellem, E., Cappellari, et al. 2011, MNRAS, 414, 2923

Kuijken, K., \& Merrifield, M. R. 1993, MNRAS, 264, 712

Kuijken, K., Fisher, D., \& Merrifield, M. R. 1996, MNRAS, 283, 543

Le Borgne, D., Rocca-Volmerange, B., Prugniel, et al. 2004, A\&A, 425, 881

Lawson, C. L., \& Hanson, R. 1995, Solving Least Squares

Problems (2d ed.; Englewood Cliffs: Prentice-Hall)

van der Marel, R., \& Franx, M. 1993, ApJ, 407, 525

Moster, B. P., Maccio, A. V., Somerville, R. S., Naab, T., Cox, T. J. 2012, MNRAS, 423, 2045

Naim, A., Lahav, O., Buta, R. J., et al. 1995, MNRAS, 274, 1107

Osterbrock, D. E., Ferland, G. J. 2006, Astrophysics of Gaseous Nebulae and Active Galactic Nuclei (2nd ed.). Univ. Science Book, Sausalito, CA

Peng, C. Y., Ho, L. C., Impey, C. D., Rix, H.-W. 2002, AJ, 124, 266

Pilyugin, L. S. \& Mattsson, L. 2011, MNRAS, 412, 1145

Press W. H., Teukolsky S. A., Vetterling W. T., Plannery B. P. 2007, Numerical Recipes: the Art of Scientific Computing (3rd ed., Cambridge Univ. Press)

Rix, H.-W., Franx, M., Fisher, D., Illingworth, G. 1992, ApJ, 400, L5

Rubin, V. C., Graham, J. A., Kenney, J. D. P. 1992, ApJ, 394, L9

Serra, P., Oosterloo, T., Morganti, et al. 2012, MNRAS, 422, 1835

Schlegel, D. J., Finkbeiner, D. P., Davis, M. 1998, ApJ, 500, 525

Sil'chenko, O. K., Moiseev, A. V., Afanasiev, V. L. 2009, ApJ, 694,1550

Sil'chenko, O. K., Moiseev, A. V., Shulga, A. P. 2010, AJ, 140 , 1462

Sil'chenko, O. K., \& Chilingarian. I. V. 2011, Astronomy Letters, 37,1

Sulentic, J. W., Verdes-Montenegro, L., Bergond, G., et al. 2006, A\& A, 449, 937

Thakar, A. R., Ryden, B. S. 1996, ApJ, 461, 55

Thakar, A.R., Ryden, B.S., Jore, K. P., Broeils, A. H. 1997, ApJ, 479, 702

Walker, I. R., Mihos, J. Ch., Hernquist, L. 1996, ApJ, 460, 121

Young, L. M., Bureau, M., Davis, T. A., et al. 2011, MNRAS, 414,940 